\title{
Application of Machine Learning Techniques for Real-Time Sign Language Detection using Wearable Sensors
}

\author{
Nazmus Saquib \\ nazmus@ucsb.edu \\ University of California, Santa Barbara
}

\author{
Ashikur Rahman \\ ashikur@cse.buet.ac.bd \\ Bangladesh University of Engineering and Technology
}

\begin{abstract}
Sign language is a method of communication primarily used by the hearing impaired and mute persons. In this method, letters and words are expressed by hand gestures. In fingerspelling, meaningful words are constructed by signaling multiple letters in a sequence. In this paper, a system has been developed to detect fingerspelling in American Sign Language (ASL) and Bengali Sign Language (BdSL) using (data) gloves containing some suitably positioned sensors. The methodologies employed can be used even in resource-constrained environments. The system is capable of accurately detecting both static and dynamic symbols in the alphabets. The system shows a promising accuracy of (up to) $96 \%$. Furthermore, this work presents a novel approach to perform a continuous assessment of symbols from a stream of run-time data.
\end{abstract}

\section{CCS CONCEPTS}

- Human-centered computing $\rightarrow$ Ubiquitous and mobile computing.

\section{KEYWORDS}

Sign Language Recognition (SLR), data glove.

\section{ACM Reference Format:}

Nazmus Saquib and Ashikur Rahman. 2020. Application of Machine Learning Techniques for Real-Time Sign Language Detection using Wearable Sensors. In 11th ACM Multimedia Systems Conference (MMSys'20), June 8-11, 2020, Istanbul, Turkey. ACM, New York, NY, USA, 12 pages. https: //doi.org/10.1145/3339825.3391869

\section{INTRODUCTION}

Sign language is a method of communication that primarily uses articulation of the hands to convey meaningful messages. It involves a simultaneous combination of hand shapes, orientation and movement of the hands, arms or body, and facial expressions. It is the most common mode of communication among the hearing and speech impaired persons.

According to the World Health Organization (WHO), over 5\% of the world population is hearing impaired [6]. This considerably large community, along with the speech-impaired community, uses sign language as the primary mode of communication. When two

Permission to make digital or hard copies of all or part of this work for personal or classroom use is granted without fee provided that copies are not made or distributed for profit or commercial advantage and that copies bear this notice and the full citation on the first page. Copyrights for components of this work owned by others than ACM must be honored. Abstracting with credit is permitted. To copy otherwise, or republish, to post on servers or to redistribute to lists, requires prior specific permission and/or a fee. Request permissions from permissions@acm.org.

MMSys'20, June 8-11, 2020, Istanbul, Turkey

(C) 2020 Association for Computing Machinery.

ACM ISBN 978-1-4503-6845-2/20/06 ..\$15.00

https://doi.org/10.1145/3339825.3391869 persons knowledgeable in sign language want to communicate with each other, they can do it conveniently. A problem arises when a person without domain knowledge of sign languages wants to communicate with a disabled person. Generally, in this type of scenario an interpreter is used. Interpreters translate sign language to oral language and vice versa. However, an interpreter might not always be available. Moreover, the use of interpreters could be very costly. A professional ASL interpreter certified by the Registry of Interpreters for the Deaf (RID) has an hourly rate of 30-35 USD [3]. Therefore, it is desirable to have an intermediary system that will translate sign language to oral language and vice versa. This work attempts to partially bridge this gap (at least) in one direction translating sign language to text.

Sign language alphabets are different for different languages. This makes automatic recognition of sign language a challenging task. For example, ASL alphabet varies widely from BdSL alphabet. Sign languages even vary from region to region. For instance, ASL is different from British Sign Language (BSL) although they share the same alphabet. Moreover, some sign languages have both single-handed and double-handed version of certain symbols. Sign languages can have a compressed form where a single word is represented by a single gesture. Sometimes instead of using a single gesture for a word sign language allows fingerspelling, which is a process of spelling out the word by using signs that correspond to the letters of the word. While using sign language, a user might choose for using a sign for the word if available, or the user might choose fingerspelling of the word. There are many words that have not been standardized in sign language dictionaries. According to [4], there are more than 150,000 words in spoken English that do not have ASL counterpart. Moreover, people's names, places, titles, brands, etc., also do not have any standardized symbols. Besides, a user might not know the exact sign for a particular word. In these scenarios, the user must resolve to fingerspelling the required words.

Traditionally, sign language is considered to be an unaided method of communication (i.e. relying on only the user's body to convey a message). However, a substantial amount of research work has been performed to aid sign language recognition using specialized tools. Traditional sign language recognition systems can be broadly classified into two categories: (i) digital image processing based systems and (ii) data glove (a glove with various sensors attached to it) based systems [7]. In the former approach, a large corpus of images of various signs is collected which are then used to train a model using supervised learning. The latter approach follows a similar trend but instead of using images, it deploys various sensors that generate necessary signals to train a classifier. In this paper, we focus on one-handed fingerspelling of 
ASL and BdSL using data glove approach. The major contributions of the paper are summarized below:

- We design and implement a data glove capable of recognizing fingerspelling in ASL and BdSL. Although commercial data gloves are extant, they might not always be readily available to the end-user. Moreover, some of the commercial gloves might need tweaking for specific languages. This work elaborately describes the construction of a data glove using sensors which are much more common than commercial gloves. The design of the glove can be changed to fit a particular language.

- We build a repository containing a data set of sensor values using the designed data glove. A crucial prerequisite in training a supervised classifier is the availability of labeled data. However, sign language data set of ASL alphabet are not publicly available, and that of BdSL alphabet does not exist at all. This work attempts to ameliorate this situation by generating a comprehensive dataset.

- We propose a system capable of recognizing sign language even under resource-constrained environment. Apart from the real-time assessment of fingerspelling, this system provides support during the data collection phase.

- Finally, we provide sign language models using data gloves. Although a number of works are found in the literature on sign language recognition using data glove, most of them provide little insight to sign language modeling, that is, determining intelligible messages from a stream of sensor data. This work proposes a methodology to infer characters and subsequently words from a continuous stream of data.

The rest of this paper is organized as follows. Section 2 presents related research works. Section 3 discusses glove construction, data collection, and the process of training a suitable classifier. Section 4 gives an overview of the system and discusses some critical design issues. Section 5 presents experimental results and the findings derived from these results. Finally, Section 6 concludes the paper with some possible directions to future works.

\section{BACKGROUND}

Over the years, sign language recognition (SLR) has been attempted in two different approaches: (i) digital image processing (DIP) based approach and (ii) data glove based approach. In both approaches, the basic idea remains the same - collecting a considerable amount of data and applying a supervised learning algorithm to classify that data. In digital image processing based systems, the data is simply pictures of various signs. On the other hand, in data glove based approach, the data is just the value of various sensors attached to the glove. Despite the basic idea being the same, there are certain differences between the two approaches that we describe next.

\subsection{DIP based Approach for SLR}

In DIP based approach, at first, a large set of images is collected. The collection process can be facilitated by either a generic camera or a specialized one such as a consumer depth camera [13]. Once the data is collected, various machine learning algorithms are applied to train a model capable of classifying images of different signs. One major drawback of this approach is the data collection procedure is inherently cumbersome, and the quality of the image might be compromised depending on the ambient light of the environment [18]. Another glaring disadvantage is it is not feasible to create a portable system using this vision-based approach. Assuming somehow the user can carry the camera with him/her, he/she still has to remain within the field of vision of the camera, thus restricting the user's distance and motion. Following the popularity of Microsoft Kinect, Dong et al. [10] attempted to use this low-cost depth camera to recognize 24 letters of ASL by using a random forest ML algorithm. Nabiyev et al. [16] applied a neural network on images of vowel characters of the Turkish language. Pandey et al. [19] proposed a methodology to segment out the hand region from the image and successively generating a feature vector of ten features. Finally, they applied a backpropagation neural network for classification. Liwicki et al. [14] worked on a dataset of 1,000 low-quality webcam videos of 100 fingerspelt words of BSL. They employed Hidden Markov Model (HMM) to interpret probable words from a stream of characters. Naoum-Sawaya et al. [17] carried out various steps to extract suitable templates from raw images, such as histogram equalization, background rejection, skin color extraction and thresholding, morphological filtering, and flood filling. After the successful generation of templates, template matching algorithm was used to classify images. Rekha et al. [21] employed K-Nearest Neighbor (KNN) and Support Vector Machine (SVM) for hybrid classification of a single signed letter. Furthermore, they proposed a lexicon-based approach to recognize fingerspelt words using HMM. Transcending the use of a simple RGB camera, Kuznetsova et al. [13] worked on real-time SLR using a consumer depth camera. The images collected from this camera were used to derive rotation, translation, and scale invariant features. They trained a multi-layered random forest to classify the feature vectors. Cui et al. [9] used Convolutional Neural Networks to map video segments to ordered gloss labels. Camgoz et al. [8] introduced Sign Language Translation (SLT) and applied Recurrent Neural Networks (RNN). This work hints at the temporal resemblance of sign language modeling to speech recognition.

\subsection{Data Glove based Approach for SLR}

A data glove has some sensors attached to it to determine various hand movement features. These features include the bending of fingers, the orientation of the hand, rotational motion of the hand, contact between two fingers, etc. Although some of the data gloves $[1,2]$ are commercially available, they are not suitable for sign language recognition due to their high cost or unavailability of specific sensors. A glove designed and created from scratch results in a cheaper product, which also provides the flexibility of incorporating only the required sensors. The cheapest glove from 5DT company costs USD 995 [2], whereas the construction of the glove designed in this work required less than USD 160. Data glove based approach removes the disadvantages of DIP based approach discussed earlier. This approach is not influenced by ambient light. The user has greater mobility, as he/she does not have to remain within the field of view of a camera. This approach is particularly convenient in a group environment where some participants know sign language while some others do not. Assuming the data glove 
is connected to some output device such as smartphones of the participants connected over Bluetooth, the user can keep on using sign language to communicate with the whole group. No other specialized device is necessary. Although quite a few research works have been performed regarding SLR using data gloves [11, 15, 20, 22], very few of them attempted to provide a complete solution - starting with the construction of data gloves to continuous detection of sign language, ensuring complete character set recognition of a particular alphabet. Patil et al. [20] attempted to classify all 26 characters of the ASL alphabet by detecting bending only. Section 3.1 explains why this is not a feasible solution. They tried to classify letters based on the ranges of flex sensor values without any specific direction on how to decide the range values. Elmahgiubi et al. [11] elaborately described a procedure of constructing a data glove capable of detecting 20 letters of the ASL alphabet. However, just like the previous work, they did not clearly how to find the boundary values to perform ranged queries. None of these works provide any method of sign language modeling, i.e. retrieving intelligible messages from a stream of data. Mehdi et al. [15] used 7-sensor glove of 5DT company to collect data and subsequently used Artificial Neural Network to classify signs. They left out two dynamic letters of the ASL alphabet and tried to detect the rest 24 letters. Although they discuss briefly sampling rate of data collection from sensors, they also do not elaborate on sign language modeling. SLARTI is a system developed at the University of Tasmania to recognize Australian Sign Language (Auslan) [22]. This system exploits the fact that signs can be described in terms of four basic manual features: (i) handshape, (ii) orientation, (iii) place of articulation, and (iv) motion. SLARTI contains four feature-extraction neural networks, one for each feature.

\section{METHODOLOGY}

In this section, we discuss several design and implementation issues of the sign language recognition system. A sign language recognition system can be subdivided into a series of discrete steps. The first step is to identify the necessary sensors and their suitable positions in the gloves to correctly capture different hand gestures. The glove is then constructed by embedding sensors at the desired positions. With the constructed glove a significant amount of data are needed to be collected which are fed to train a Machine Learning (ML) model to classify the captured data. A detailed study of the dataset might reveal whether a further modification to the glove and relative positions of the sensors is necessary or not. If a modification is required, all or a few of the previous steps need to be repeated. Once a suitable model is trained, sign language modeling, that is, determining intelligible messages from a stream of sensor data starts.

\subsection{Data Glove Construction}

Several options are available for the base of the data glove. A normal woolen glove can be used as the base. However, as woolen gloves tend to be roomy and do not fit tightly around the hands, other types of gloves are preferable. Lycra gloves, wicketkeeper's inner gloves, etc. are all good candidates. Surgical gloves can be used too, but as these tend to fit too tightly around the hand, it might become cumbersome to wear and take the glove off repeatedly with all the

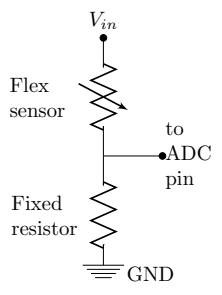

Figure 1: Voltage divider circuit.

sensors attached to it. As the same glove would be used by people with varying hand sizes, an average-sized glove is preferred.

Detection of Bending. The primary criteria to classify symbols in ASL and BdSL (or any other sign language) is the bending of the individual fingers. To detect this bending a type of resistive sensor called flex sensor can be used. Flex sensors are made of a material which changes its resistance upon bending. In particular, the resistance increases as the flex sensors are bent. Five flex sensors are required to detect the bending of five different fingers. The flex sensors are attached to the back of each finger. As microcontrollers cannot measure resistance directly, a voltage divider circuit is required. Interestingly, the exact resistance value of a flex sensor at a certain instance is not required, rather a measure of the change in resistance serves the purpose. The more the sensor is bent, the more its resistance increases. Therefore, by simply observing the relative changes in resistance value, it can be inferred whether the sensor is straight or bent. The voltage divider circuit shown in Fig. 1 has been used. When the flex sensor is bent, its resistance increases, which results in lower voltage in Analog-to-Digital Converter $(A D C)$ pin. If the flex sensor is subsequently straightened, its resistance decreases, which results in higher voltage in ADC pin. Detection of Contact. Although just by determining the relative bending of each finger several symbols can be classified, there exist a few symbols that cannot be classified solely based on this information. For example, the ASL characters ' $M$ ', 'N', and ' $T$ ' have similar bending of the fingers. In case of ' $M$ ', the thumb goes between the little finger and the ring finger, in case of ' $N$ ' the thumb goes between the ring finger and the middle finger, and in case of ' $\mathrm{T}$ ' the thumb goes between the middle finger and the index finger. To differentiate these letters the contact information between the thumb and other fingers is needed. Similarly, the letters ' $R$ ', ' $U$ ', and ' $V$ ' also have similar bending of individual fingers but vary in contact points.

The most viable option to capture contact information is to imagine the contact sensors simply as buttons. If there is a contact i.e. the button is $\mathrm{ON}$, then a particular signal level is generated. If there is no contact i.e. the button is OFF, then another level of signal is generated. Instead of an actual button, the two ends of the button can easily be simulated by conductive surfaces. A cheap and readily available solution could be obtained from aluminum foils used as chocolate wrappers as shown in Fig. 2. One drawback of using such foils is that over time they tend to form creases at certain points due to bending and lose conduction at the points of crease formation. Other than aluminum foils, conductive fabrics or conductive threads can also be used as contact sensors. The final implementation of the glove in this work has used conductive fabric. Detection of Orientation and Motion. Apart from the bending 


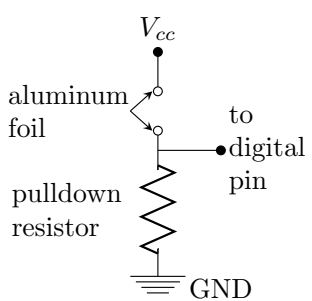

Figure 2: Simulating button using aluminum foil. Table 1: Hand size of participants.

\begin{tabular}{|c|c|c|c|}
\hline User ID & Length & Circumference & User comment \\
\hline 1 & 7.0 & 6.5 & Slightly roomy \\
\hline 2 & 6.5 & 6.1 & Too large \\
\hline 3 & 7.2 & 7.2 & Too tight \\
\hline 4 & 6.9 & 6.8 & Comfortable \\
\hline 5 & 7.0 & 7.0 & Perfect fit \\
\hline
\end{tabular}

of fingers and contact between two fingers, another feature that is sometimes required is the orientation of the hand. For example, the ASL letters ' $H$ ' and ' $U$ ' have similar bending and contact, the only difference is their orientation. Similar observations can also be made for the letter pairs $\langle G, Q\rangle$ and $\langle K, P\rangle$. One last feature required to differentiate certain characters is motion. Although most characters in ASL and BdSL are static, very few are dynamic, meaning motion is involved when they are signed. For example, the ASL letters ' $J$ ' and ' $Z$ ' are signed by drawing the letters in the air with a finger. To detect orientation and motion, an accelerometer and gyroscope unit can be used. The best place to attach the unit would be back of the hand, a few centimeters below the wrist.

\subsection{Data Collection}

Data has been collected from five users with varying hand sizes using the same glove. Table 1 shows the hand sizes and comments for the five participants. To measure the hand size, two metrics have been used - one is length, another is circumference. To facilitate the process of data collection and later on real-time evaluation, a desktop application was developed. Each user gave data in two sessions for each language (ASL and BdSL). In each session, the user gave 500 data for each character. Therefore, for each character, there are 1000 data points from each user. There are some common signs between the ASL and BdSL alphabet, they have been collected once. In each session, a user gave data for one character in five iterations. An iteration consists of the user holding the sign for ten seconds. Data was collected at $100 \mathrm{~ms}$ interval, resulting in 100 data in one iteration. Between successive iterations, the user straightened his/her hand once and then made a fist with his/her hand once before moving onto the next iteration. This has been done so that the previous iteration does not have any effect on the next iteration. This ensures variability in data which would be observed when a user is signing different letters continuously. In the case of dynamic signs, the stationary state of the sign has been recorded. As 'I' and 'J' have the same static configuration of hand, the dataset contains the data of 'I' but not that of 'J', 'J' being the dynamic one.
Each feature vector consists of sixteen features. The first five features, namely thumbFlex, indexFlex, middleFlex, ringFlex, and littleFlex, correspond to the values of five flex sensors attached to five fingers. These are required to detect the bending of each of the fingers. The next five features are for five contact sensors placed at various locations of the hand. The locations were chosen by studying the symbols through an iterative approach to data collection and classification. A contact sensor was placed at a suitable location only if more than one character showed similar bending but varied in contact. Five places were identified to be enough to distinguish among the characters of ASL/BdSL. The five features corresponding to these places are indexFront, middleFront, middleSide, ringSide, and littleSide. The next three features correspond to the X, Y, and $\mathrm{Z}$ components of the accelerometer. It was observed that certain pairs of characters have similar reading for flex sensors and contact sensors. However, they differ in orientation. An accelerometer was used to detect this difference in orientation. The three features next to that correspond to the $\mathrm{X}, \mathrm{Y}$, and $\mathrm{Z}$ component of the gyroscope. There are a few dynamic characters in ASL and BdSL. The motions involved in these dynamic characters can be detected using a gyroscope. The last three columns in the dataset correspond to the letter being signed i.e. the label, the user ID, and the session ID for that particular user respectively. As the difference of data over users and sessions has been studied in detail in Section 5.1, user ID and session ID have been included in the dataset. This amounts to a total of nineteen columns in the dataset, among which sixteen are features. The full dataset can be accessed at [5].

The necessity of various sensors described in Section 3.1, and in turn the selected features, can be more succinctly understood with the help of certain features observed together. For example, ' $U$ ' and ' $V$ ' have similar bending for all the fingers and thus are not separable based solely on flex sensor values. However, the index finger and the middle finger come in contact while signing ' $U$ ', but not while signing ' $V$ '. That is, the contact sensor positioned between these two fingers give a value of 1 for ' $U$ ' but 0 for ' $V$ ', while the flex sensor values remain similar for both the characters. This phenomenon is demonstrated in Figure 3. The plots were generated by keeping all of the fingers straight and apart from each other for some time, then moving on to the symbol of a character and holding it for some time, followed by a fully straight hand again. Similarly, the use of accelerometer can be justified with cases such as ' $\mathrm{H}$ ' and ' $\mathrm{U}$ ' as shown in Figure 4. In the case of these two letters, the bending of the fingers is similar but the orientation is different. This difference in orientation is apparent from the X component of the accelerometer reading. Finally, the role of a gyroscope in detecting motion for dynamic characters such as ' $Z$ ' is evident from Figure 5. The beginning and the ending of the plot corresponds to the static ' $Z$ ' symbol, while the middle portion corresponds to the $z$-shaped motion. As can be seen from the figure, the flex sensor values remain similar throughout the period. However, a significant amount of fluctuation is observed in gyroscope reading during the motion. The points corresponding to the time when first horizontal motion, the diagonal motion, and the second horizontal motion was detected have been labeled. 


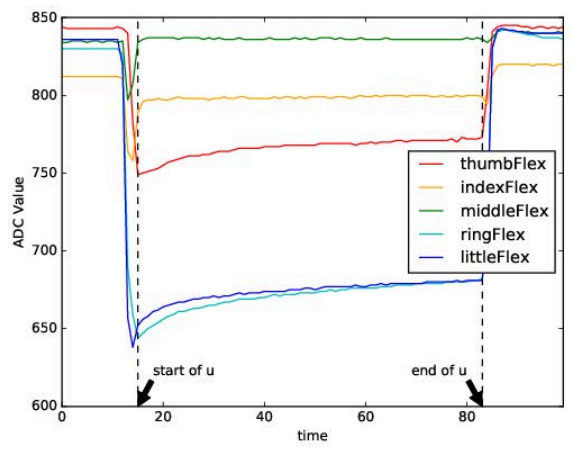

(a) Linechart of flex sensors for ' $U$ '.

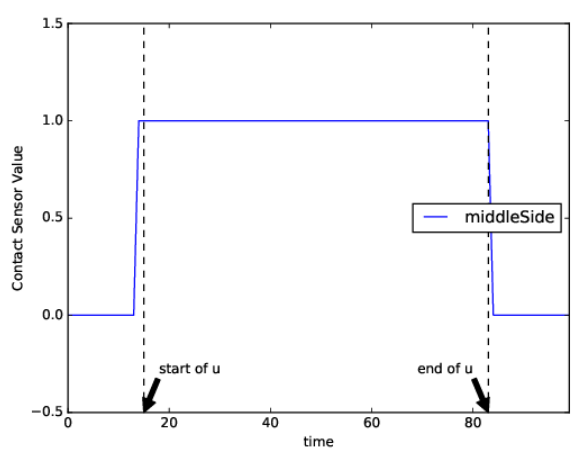

(c) Linechart of middleSide contact for ' $U$ '.

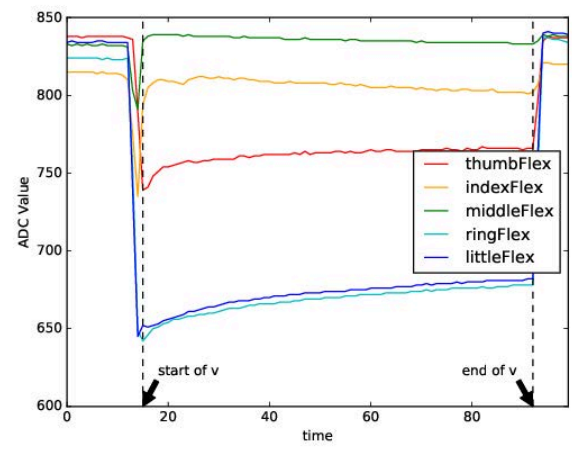

(b) Linechart of flex sensors for ' $V$ '.

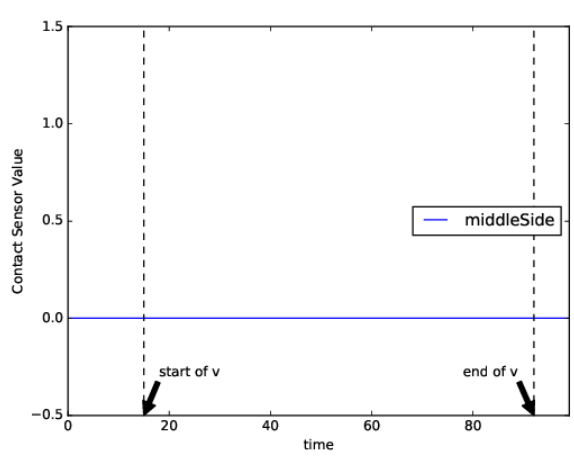

(d) Linechart of middleSide contact for ' $V$ '.

Figure 3: Linecharts of flex sensors and the contact sensor between the index finger and the middle finger for ' $U$ ' and ' $V$ '.

\subsection{Training a Classifier}

Once data has been collected, the next step is to train a suitable classifier. As the model should be able to work in a resource-constrained environment, not all classifiers are suitable. For example, lazy learners like KNN are out of consideration. On the other hand, neural networks have an added advantage that the model can be trained on a different processor and only the weights can be simply loaded into the microcontroller. Due to this fact, if a neural network-based classifier requires heavy computation during the training phase it is not a concern. But if the testing phase requires heavy computation, that classifier should be avoided.

Although at first glance one might assume that there should be a single classifier for the two languages (ASL and BdSL), in reality, two different classifiers are required. This is because there are eleven overlapping symbols between the two languages. There is an option in the system for the user to choose the desired language.

While training, the classifier is trained based on the values from the five flex sensors only. In other words, an initial classification is made based on the bending of the fingers. This essentially puts a feature vector in a cluster, and if the cluster contains more than one letter, a rule-based classification is employed. The exact rule can be found out by simply fitting a decision tree on the values of contact sensors and accelerometer. For example, the letters ' $V$ ' and ' $U$ ' have a similar type of bending of the fingers. So ' $V$ ' can be classified as ' $U$ ' and vice versa by the classifier, i.e., the classifier puts it in a cluster containing two letters: ' $V$ ' and ' $U$ '. But a contact sensor between the index and middle fingers allow the rule-based classifier to distinguish between these two characters. A block diagram of the classification subsystem is presented in Fig. 6.

It is worth noting that instead of multi-level classification, an end-to-end classification approach can be employed too, i.e., feeding all of the data from flex sensors, contact sensors, and accelerometer. However, the former approach was employed in the initial implementation and later kept unchanged for a few reasons. Firstly, the glove construction and the training steps are closely coupled in this work. When it was observed that flex sensors alone were not sufficient for classification, only then the other sensors were incorporated in the glove. As the contact sensors provide a value of 0 or 1 and the accelerometer mostly provides a value within $[-1,1]$, intuitively it made more sense to pass the sensor readings through a decision tree to break any sort of tie. Secondly, this approach, in general, uses a minimal amount of data during computation. At first, only the flex sensor values are used for computation during classification, data from other sensors are used only when required. Finally, it was observed that an end-to-end classifier did not affect the accuracy considerably. There was a very slight deterioration in accuracy percentage for an end-to-end classifier, the difference being in the hundredths place. Once a classifier has been trained on the collected data, it can be deployed in a production environment. 


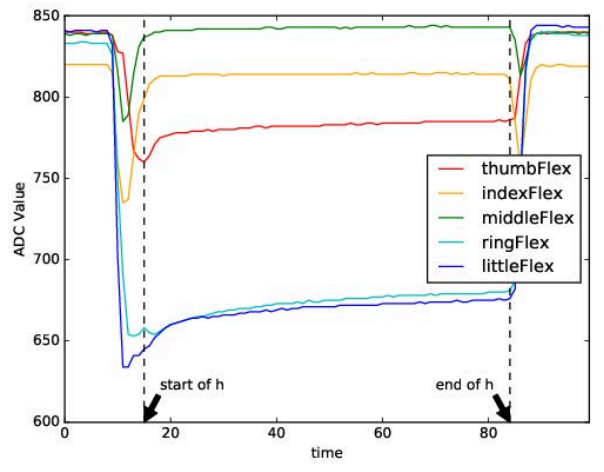

(a) Linechart of flex sensors for ' $H$ '.

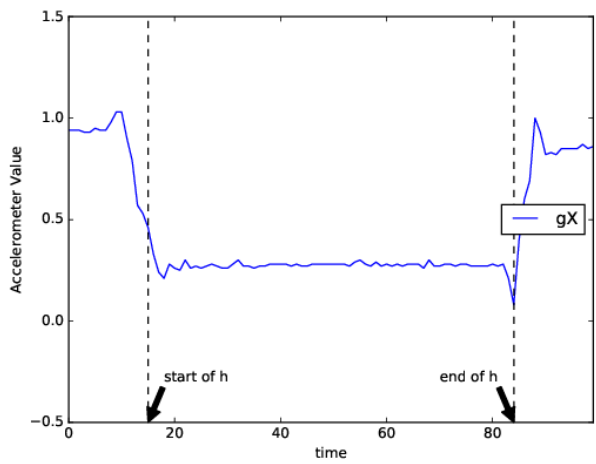

(c) Linechart of $\mathrm{gX}$ for ' $\mathrm{H}$ '.

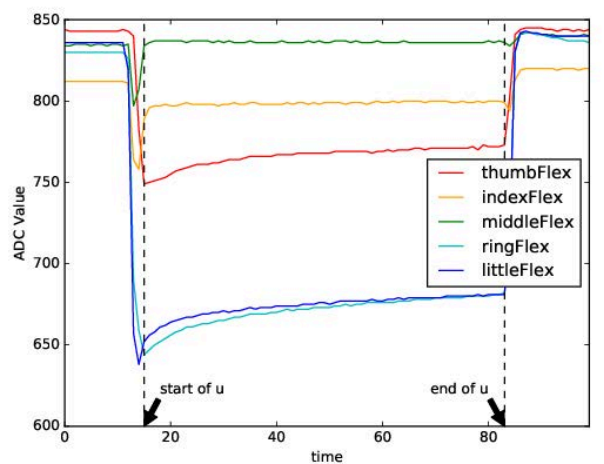

(b) Linechart of flex sensors for ' $U$ '.

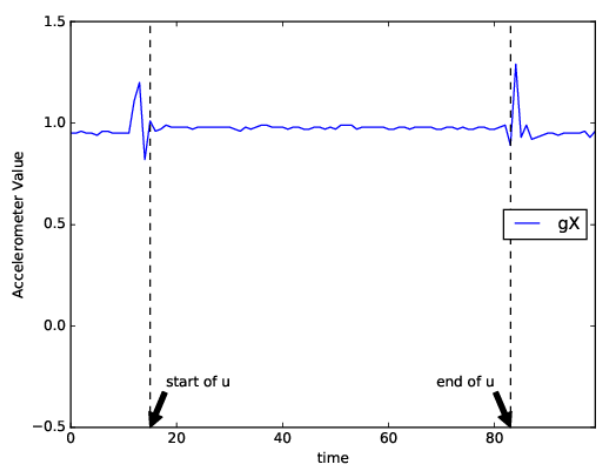

(d) Linechart of $\mathrm{gX}$ for ' $\mathrm{U}$ '.

Figure 4: Linecharts of flex sensors and $X$ component of accelerometer for ' $H$ ' and ' $U$ '.

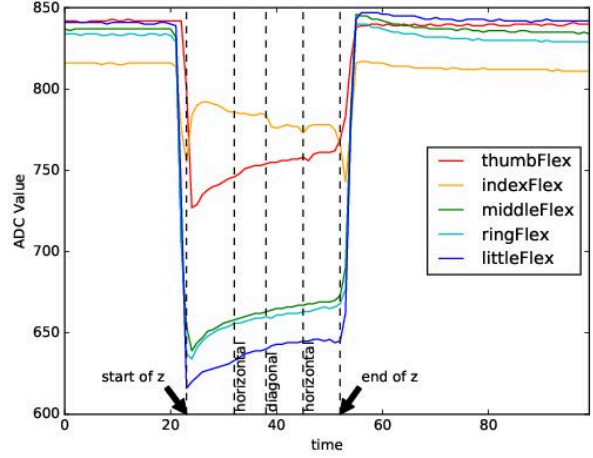

(a) Linechart of flex sensors for ' $Z$ '.

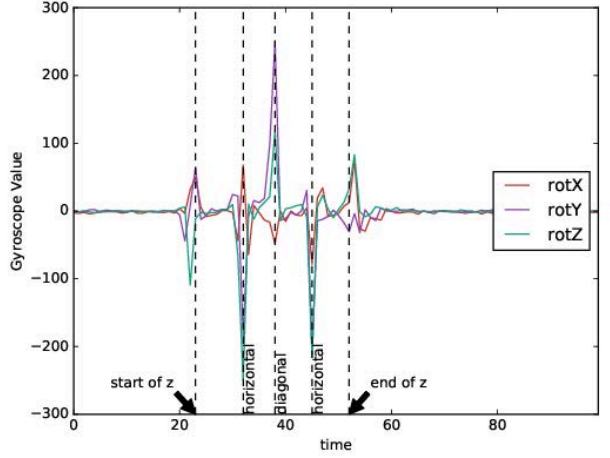

(b) Linechart of gyroscope values for ' $Z$ '.

Figure 5: Linechart of values from flex sensors and gyroscope for ' $Z$ '.

\section{SYSTEM DESIGN AND TUNING}

This section discusses the design issues of the developed system. Section 4.1 discusses interfacing among the various components of the system. Section 4.3 presents a simple technique for sign language modeling, i.e. the process of forming intelligible messages from a stream of sensor values. The developed technique is capable of filtering out the transitional noise observed when the user moves from letter to letter. A suitable Machine Learning algorithm has been chosen from four after a comparative study for the final deployment of the system. The four algorithms are Artificial Neural 


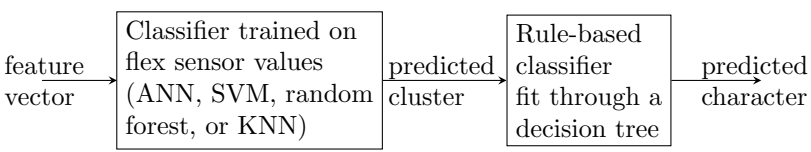

Figure 6: Block diagram of the classification subsystem.

Network (ANN), Support Vector Machine (SVM), Ensemble Learning (Random Forest), and K-Nearest Neighbor (KNN). Section 4.5 discusses suitable parameter selection for these algorithms.

\subsection{Physical Connectivity}

The constructed glove is interfaced with an Arduino Mega microcontroller board via circuitry concised in a veroboard. The sensor data collected by the microcontroller is then sent over to a computer via the USB port. A variant of this setup might be to altogether get rid of the computer and perform all the functionalities in the microcontroller. That is also possible but would require some extra components. For data collection purpose the microcontroller would require a Micro SD card/SD card breakout board along with the card, and for display purposes, it would require a display module. Alternatively, the microcontroller could communicate to a smartphone via Bluetooth.

A desktop application was developed in Java to facilitate data collection and real-time assessment. A labeled diagram of the realtime assessment window is presented in Fig. 7. The line chart shows the ADC values as affected by the bending/straightening of flex sensors. There are five lines for each of the flex sensors attached to the five fingers. Just below the line chart are five indicators representing whether the contact sensors are in contact or not. Next, the accelerometer and gyroscope readings are dumped in a tabular fashion. At the very bottom, there is a serial display which shows a set of sensor values once the test button situated at the top row is pressed. The window contains a couple of dropdowns to select the language and character. The latter dropdown is used only in data collection mode, but not in real-time assessment mode. The $\log$ button is also exclusively used in data collection mode. On the top-right, there is an image that contains the letter being signed at that instant. Just below that there is a window showing the stream of characters as classified by the trained classifier. The underscores represent space in this display. An explicit character has been used to represent space so that it is obvious exactly when the system introduces a pause after the last character being recognized. If a user continues to give sign without sufficient pause these will not be displayed, following the convention of fingerspelling. This window clears itself once it gets full.

\subsection{Detection of Dynamic Symbols}

Both ASL and BdSL alphabets have a few dynamic letters. A simple way to detect dynamic symbols is to map them to finite state machines (FSM). As ' $Z$ ' is the most complex among all of the dynamic symbols, the detection of ' $Z$ ' is explained in detail. Other dynamic symbols can be similarly mapped to corresponding state machines. To express ' $Z$ ', a user needs to first make the static ' $Z$ ' symbol. While holding this symbol, the user needs to make one rightward

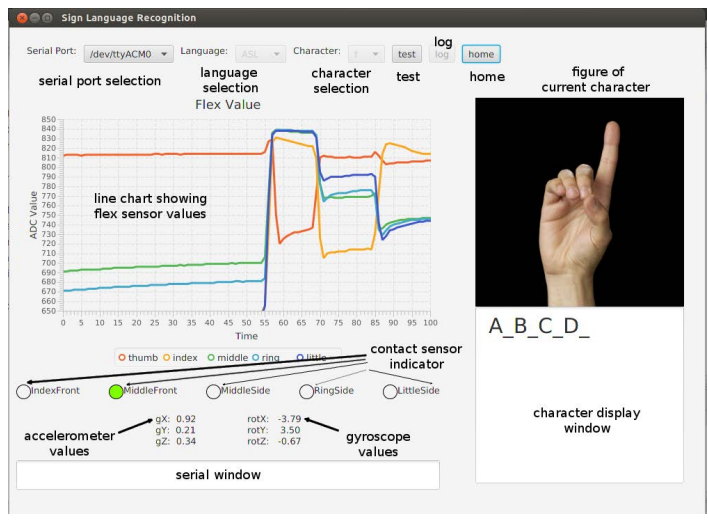

Figure 7: Labeled real time assessment window.

movement, followed by a diagonally downward movement, then finally another rightward movement. One movement must follow the previous one within a certain time limit $t$, i.e. everything is done in one swift motion. Fig. 8 shows a finite state machine to detect ' $Z$ '. Here the state $Z$ ' represents any symbol other than ' $Z$ ', state $Z_{s 1}$ represents the static ' $Z$ ' symbol, state $Z_{s 2}$ represents ' $Z$ ' up to first rightward movement, state $Z_{s 3}$ represents ' $Z$ ' up to diagonally downward movement, and finally, $Z$ represents the successful detection of ' $Z$ '. The rightward and diagonally downward movements can be detected by studying the current and a few prior sampled values of the gyroscope.

\subsection{Sign Language Modeling}

Data is collected from the sensors at an interval of $100 \mathrm{~ms}$. At this rate, a classifier makes a classification as soon as the sensor data is fed to it. But right after the classifier predicts that the current symbol represents a character $X$ (here $X$ is a placeholder), it is not displayed by the system. Rather the system would wait till it sees a number equal to a MINIMUM_THRESHOLD of consecutive $X \mathrm{~s}$. This has been done to remove the effect of noise during transitions from one letter to another. The value of this MINIMUM_THRESHOLD might again depend on the speed of an individual. While using fingerspelling, a space or division between words is represented by holding a symbol longer than usual. Therefore, another parameter, MAXIMUM_THRESHOLD, has been introduced. If a symbol is continuously observed for more than or equal to this number, a space is introduced by the system. An obvious question is how to show double letters. In fingerspelling, a double letter is shown by giving a slight bump of the hand, or a slight motion to the side. This bump/motion can be detected with the help of a gyroscope. So if a bump is observed before MAXIMUM_THRESHOLD, the system could display a double letter. However, it has been observed that the readings shown by gyroscope while forming a bump correspond to similar readings shown during certain transitions from character to character. This resulted in the system to show double characters when in fact there was only a transition. Hence this feature was left out. The modeling algorithm keeps track of a variable, COUNTER, which represents the number of contiguous steps the current character has been predicted. When the current prediction $X$ is observed to be different than the previous prediction $X^{\prime}$, the COUNTER is 


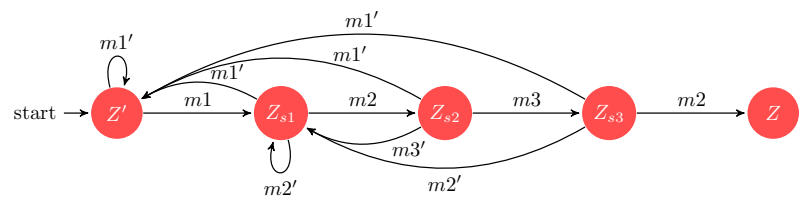

$m 1:$ the ' $\mathrm{z}$ ' symbol

$m 2$ : rightward movement while holding $m 1$ within an interval $t$ of previous state

$m 3$ : diagonally downward movement while holding $m 1$ within an interval $t$ of previous state

$m 1^{\prime}$ : any symbol other than ' $\mathrm{z}$ '

$m 2^{\prime}$ ': any movement other than $m 2$ while holding ' $\mathrm{z}$ '

$m 3$ ': any movement other than $m 3$ while holding ' $\mathrm{z}$ '

Figure 8: FSM to detect ' $Z$ '.

reset to one. In the current system, to represent a double letter the user needs to deliberately create some transitional noise in order to reset the COUNTER. Fig. 9 shows a simulation of this sign language modeling in action. Here the MINIMUM_THRESHOLD is set to two and the MAXIMUM_THRESHOLD is set to four. When the first ' $\mathrm{A}$ ' is observed, the COUNTER is set to one. The second ' $\mathrm{A}$ ' increases the COUNTER to two. As the COUNTER is now equal to the MINIMUM_THRESHOLD, an 'A' is displayed (step 2). Next, another ' $A$ ' comes up, increasing the COUNTER to three. Then another ' $A$ ' increases the COUNTER further to four. As the COUNTER is equal to the MAXIMUM_THRESHOLD, a space is inserted in display (step 4). Another ' $A$ ' after that increases the COUNTER to five. Now a ' $C$ ' comes up. As the current prediction ' $C$ ' is not equal to the previous prediction 'A', the COUNTER is reset to one. Next, a 'B' comes up, resetting the COUNTER to one again. Another 'B' increases the COUNTER to two, making it equal to MINIMUM_THRESHOLD, so a ' $\mathrm{B}$ ' is displayed (step 8). It is worth noting that the transitional noise 'C' was filtered out by using this simple mechanism.

\subsection{System Integration}

Figure 10 provides an overview of the full system. Data is read from the glove at a definite sampling rate by the processing unit. The processing unit contains a previously trained model of a classifier and an implementation of the sign language modeling algorithm described in Section 4.3. The classifier is trained using previously collected labeled data as described in Section 3.3. If the system is operating in data collection mode, the processing unit sends the data to a computer (which can also act as a display unit) for storage and the successive training of a classifier. If the system is operating in real-time classification mode, the processing unit performs two extra steps before sending data to the display unit. Firstly, it is going to classify the set of sensor values just read in into one of the characters using the pre-trained model of a suitable classifier. Secondly, it is going to input the classified character to the algorithm of sign language modeling. The sign language modeling algorithm outputs the character to be displayed depending on the recent few classified characters as described in Section 4.3. Thus, at first, a stream of sensor values sampled at a definite rate gets classified by a pre-trained classifier to generate a stream of characters. Then the sign language modeling algorithm dictates which character should be displayed depending on that stream of characters. Finally, the display unit shows the actually recognized character.

\subsection{Sensitivity Analysis}

Each of the studied algorithms has one or more tunable parameters. To fix a parameter of an algorithm, the data were randomly split into $80 \%$ train data and $20 \%$ test data. It was ensured during the split that the proportions of data from user $X$ for character $Y$ were equal for all $X$ and $Y$. Then the same algorithm was run over the split by varying the value of the parameter. The value corresponding to the highest accuracy was fixed for that parameter.

In the case of ANN, a single hidden layer was used. Studying the effect of increasing the number of layers was ruled out considering the involved time and space complexity. It was observed that accuracy increased steadily as the number of nodes in hidden layer was increased up to 7 (24) for ASL (BdSL). After that, the accuracy oscillates around a certain mean. Four activation functions were studied - tanh, logistic, identity, and relu. It was observed that the logistic function showed the best accuracy for both ASL and BdSL. In the case of SVM, Radial Basis Function (RBF) showed better accuracy compared to linear, poly, and sigmoid. In the case of random forest, the accuracy increased steadily as the number of stumps was increased up to 40 (32) for ASL (BdSL). After that, the accuracy oscillated erratically. In the case of KNN, the accuracy did not considerably change after increasing the value of $\mathrm{K}$ beyond one for ASL. On the other hand, the accuracy varied wildly for BdSL, exhibiting a peak for $\mathrm{K}=200$. A summary of the different parameters for the four algorithms is presented in Table 2.

\section{EXPERIMENTAL RESULTS}

Experiments were conducted on the dataset to understand the composition of data and to analyze the performance of various algorithms on the data. As the data was collected from multiple users in different sessions, one question of interest was whether the data between any two users (or two sessions of a user) are statistically significantly different. This question is addressed in Section 5.1. At first take it might seem like a simple range-based query over the flex sensor data might be sufficient to classify the letters. Section 5.2 elaborates why range-based query does not give good results. Section 5.3 discusses the relative nonuniformity in bending of fingers over different users. Finally, Section 5.4 compares the performance of four algorithms over the dataset.

\subsection{Difference of Data over Users and Sessions}

To answer the question of whether the data between any two users (or two sessions of a user) are statistically significantly different, Wilcoxon Signed Rank Test was conducted at 0.05 significance level. It was observed that all pairs of users showed statistically significant difference in at least one of the five flex sensors. Among the five users, three showed a statistically significant difference in at least one of the five flex sensors between different sessions. Among the two users whose data did not exhibit statistically significant difference over sessions, one user opined that the glove fit perfectly whereas the other user stated the glove was comfortable. On the other hand, the greatest difference over sessions was observed for the user with the smallest hand size. This conforms to the intuitive observation that every time a user with a small hand size wears the glove, the sensors tend to sit on his/her hand a bit differently. Therefore, the readings would be different each time. Conversely, 
MINIMUM_THRESHOLD $=2 \quad$ MAXIMUM_THRESHOLD $=4$

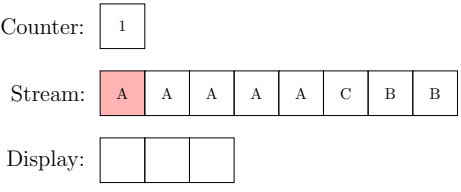

(1)

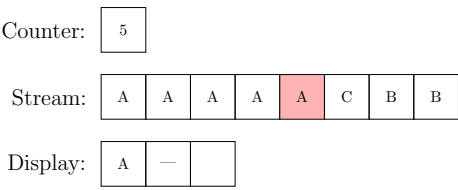

(5)
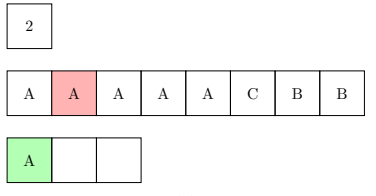

(2)

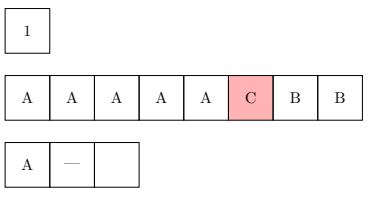

(6)

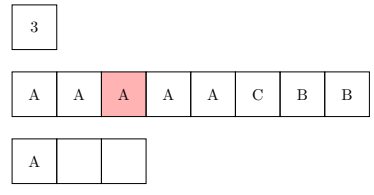

(3)

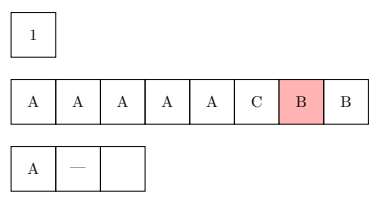

(7)

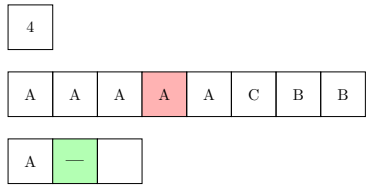

(4)

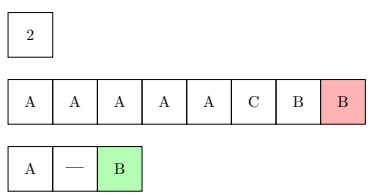

(8)

Figure 9: A simulation of sign language modeling.

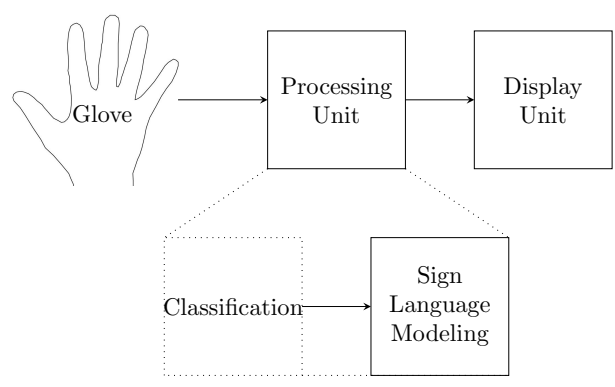

Figure 10: Block diagram of the full system. Table 2: Chosen values of different parameters.

\begin{tabular}{|l|l|l|l|}
\hline Algorithm & Parameter & ASL & BdSL \\
\hline ANN & \#nodes in hidden layer & 7 & 24 \\
\hline ANN & activation function & logistic & logistic \\
\hline SVM & kernel function & RBF & RBF \\
\hline Random Forest & number of stumps & 40 & 32 \\
\hline KNN & no. of neighbors $K$ & 3 & 200 \\
\hline
\end{tabular}

a user with a good fit will experience similar positioning of the sensors over different sessions. As a result his/her data would not exhibit significant difference over different sessions.

\subsection{Infeasibility of Range-Based Query}

The classification was first attempted through a range-based query. However, it was observed that not all characters can be differentiated by simply observing the range of flex sensor values. Fig. 11 shows boxplot of flex sensor values on the middle finger for letters ' $\mathrm{B}$ ', 'S', and ' $\mathrm{X}$ '. A brief look at the plot reveals that there is a clear distinction between the range of values for ' $B$ ' and ' $S$ '. The same can be said for the pair ' $B$ ' and ' $X$ '. However, ' $S$ ' and ' $X$ ' have a considerable amount of overlap over their full range. Similar trends can be observed for the other flex sensor values. Moreover, ' $\mathrm{S}$ ' and ' $\mathrm{X}$ ' are such a pair that cannot be differentiated with the help of existing contact sensors, they must be differentiated with the help of the flex sensor values. Therefore a more elegant solution is required for classification. Later classifications were attempted using various

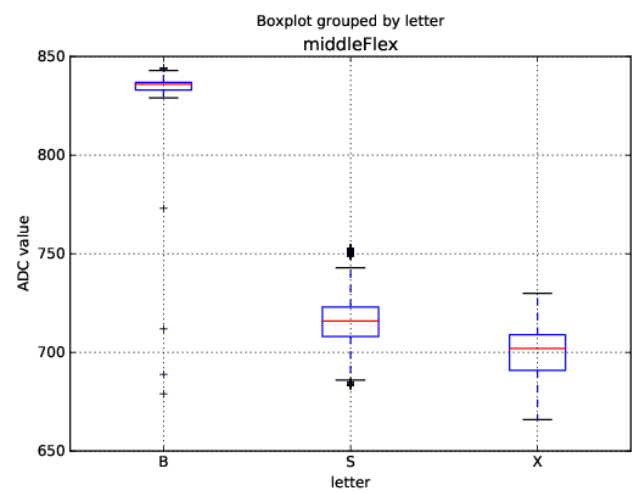

Figure 11: Boxplot of middle flex values for ' $B$ ', ' $X$ ', and ' $S$ '.

Machine Learning algorithms that exhibited superior performance.

\subsection{Nonuniformity in Relative Bending}

One interesting observation of this study is that the same trend in relative bending of the fingers of different users can not be observed for all letters, thus rendering an initial calibration ineffective. Fig. 12a and Fig. 12b shows the line charts of mean values of flex sensors for all five users for the letters ' $A$ ' and 'P' respectively. From the line chart of 'A', it is evident that the little finger of User 4 experiences less bending compared to that of other users. Intuitively, it might seem like this should be the case for all other letters. However, the line chart of 'P' reveals one of the many counterexamples. Here it is observed that the little finger of User 4 actually experiences the second most bending among the five users. Therefore, no general comment can be made regarding the relative bending of different users.

\subsection{Performance of Different Algorithms}

A K-Fold Cross-Validation was performed on the dataset by taking ten folds. The average accuracy over the ten iterations is presented in Table 3. It can be observed from the presented accuracies that the average accuracy of random forest and KNN are better than SVM. The performance of ANN is slightly below that of random forest 


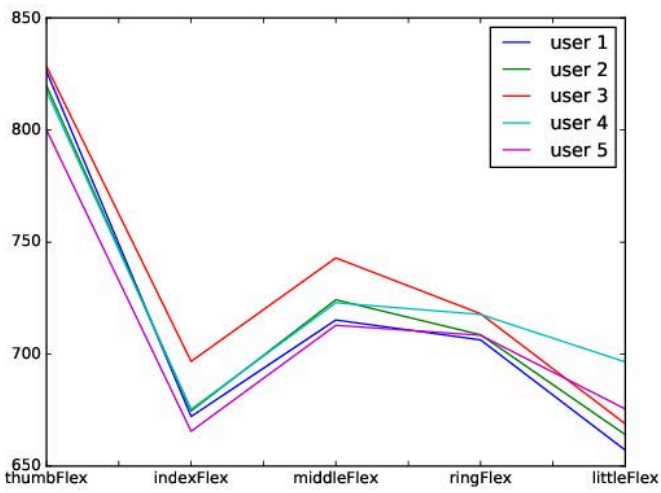

(a) Linechart for ' $A$ '.

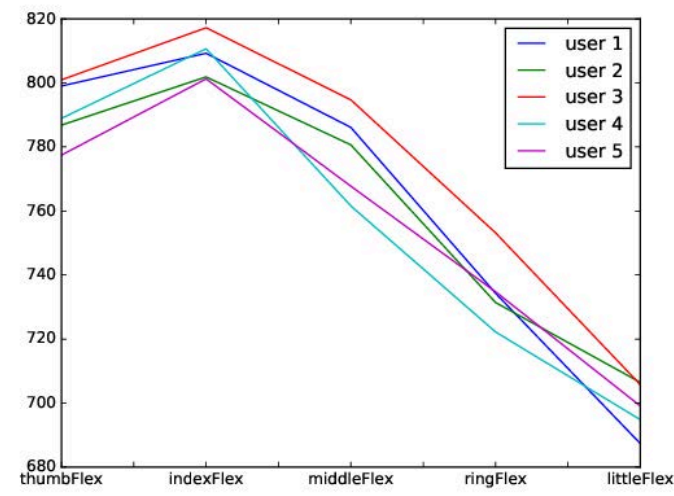

(b) Linechart for ' $P$ '.

Figure 12: Linecharts of mean values of flex sensors of different users for ' $A$ ' and ' $P$ '.

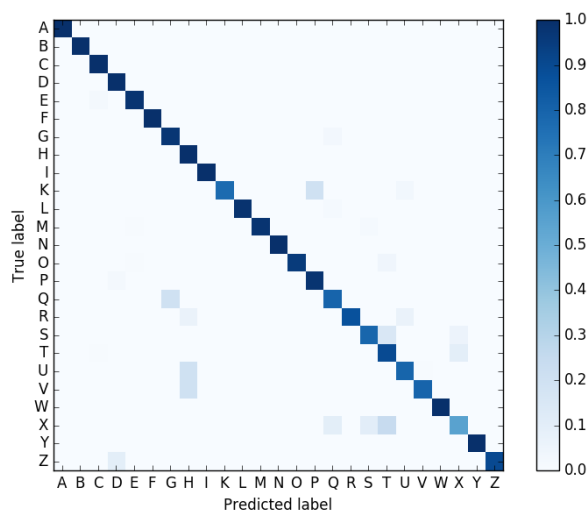

Figure 13: Confusion matrix for ANN run on a random 80-20 split of ASL dataset.

and $\mathrm{KNN}$. However, as $\mathrm{KNN}$ is a lazy learner, it is not suitable for embedded environments. Random forest could be deployed in an embedded environment, but between a trained model of ANN and a trained model of random forest, ANN has been found to be faster Therefore, ANN might be taken as the overall best choice. Fig. 13 and Fig. 14 shows the color-coded confusion matrix resulted from running ANN on a random 80-20 split of ASL and BdSL dataset respectively.

A trained ANN model was deployed in the final system. It was observed that the final accuracy of the system for fingerspelling recognition was around $96 \%$, closely following the performance of the classifier over the collected data. However, the two threshold values required by the sign language modeling algorithm had to be manually tweaked for different users; as otherwise spaces were being introduced for comparatively slower users before they expected, and some letters were missed by the system for comparatively faster users. As future work, a calibration phase can be added to automatically set the threshold values for different users.

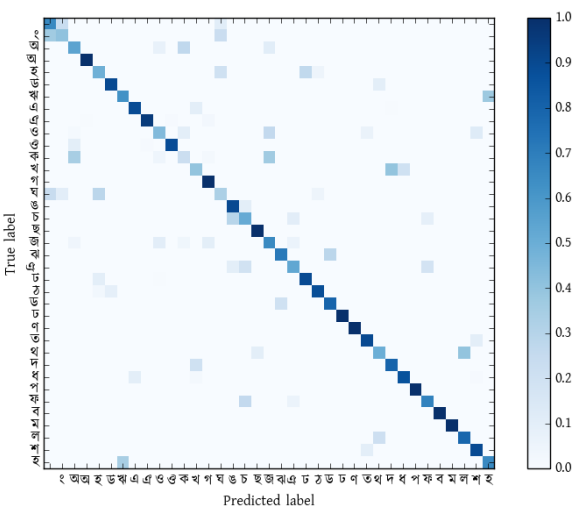

Figure 14: Confusion matrix for ANN run on a random 80-20 split of BdSL dataset.

Table 3: Average accuracy after 10-Fold Cross Validation.

\begin{tabular}{|l|l|l|}
\hline \multirow{2}{*}{ Algorithm } & \multicolumn{2}{|c|}{ Accuracy (\%) } \\
\cline { 2 - 3 } & ASL & BdSL \\
\hline KNN & 96.1448 & 93.8643 \\
\hline Random Forest & 96.1352 & 96.3422 \\
\hline ANN & 95.8784 & 93.4368 \\
\hline SVM & 94.916 & 92.1859 \\
\hline
\end{tabular}

\subsection{Classification of Non-Standard Gestures}

Currently, it is possible to confuse the system with certain nonstandard gestures which might look like one character to the naked eye but is actually classified as another character by the system. The main reason behind this can be thought of as the non-availability of sensor values corresponding to these gestures during the training phase of the classifier. However, it is not feasible to collect data for all possible non-standard gestures. This problem can be solved to some extent by generating some synthetic data. Generation of 


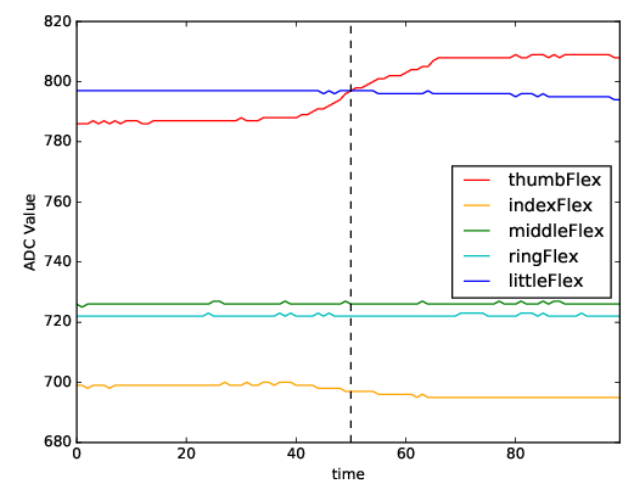

Figure 15: Flex sensor values corresponding to gestures for 'I', gradually followed by deformed gestures.

synthetic data and its subsequent use in training a classifier has been extensively studied in the literature $[12,23,24]$. In the future, this strategy can also be employed for this work. It has also been observed that in many cases the system outputs the correct result even when the gestures are deformed to some extent, and starts to show a different result only when the gesture becomes ineligible to the naked eye too. As an example of a confusing case of nonstandard gesture, flex sensor values corresponding to some gestures of the letter 'I' have been presented in Figure 15. In the case of 'I', all the fingers except the little finger are bent, the thumb is not bent fully but bent enough to cross in front of the index finger. The first half or so of the plot was obtained from this standard gesture. After that, the thumb was gradually moved to the side so that it lost contact with the index finger but still looked close to it. In this condition, the gesture might still look like 'I' to the naked eye. However, the system recognized this gesture as ' $Y$ '. In a standard gesture of ' $Y$ ', the thumb is separated from the index as far as possible. The exact position from where the system started to detect ' $\mathrm{Y}$ ' has been marked with a dashed vertical line in the plot.

\subsection{Comparison with Previous Works}

Comprehensive comparison among the previous works on sign language recognition is presented in Table 4. Most of the previous works were on signed letter recognition, whereas Naoum-Sawaya et al. [17] and Vamplew et al. [22] attempted signed word recognition i.e. words that can be expressed using a single gesture. The former used DIP based method whereas the latter used glove based method. The more complex case of sign language modeling using DIP based method was covered by Liwicki et al. [14] and Rekha et al. [21]. However, to the best of our knowledge, sign language modeling using data gloves has not been studied so far, which this work does. Moreover, the variability in data collected from different users has not been studied extensively by the previous works. On the other hand, this work makes a detailed observation of this aspect. Although some works reported recognition of dynamic characters, they did not clarify the underlying mechanism. This work provides a detailed explanation of an FSM based method of dynamic character recognition.
Among the works based on DIP, the work by Kuznetsova et al. [13] showed the best accuracy of $97.55 \%$. However, they left out the two dynamic characters of ASL, 'J' and ' $Z$ '. Nabiyev et al. [16] showed an accuracy of $96 \%$ on only the six vowels of the Turkish alphabet. Although some works based on DIP included the dynamic characters $[7,18,19]$, no clarification was provided regarding how the recognition of dynamic characters was achieved. Liwicki et al. [14] worked on double-handed fingerspelling in BSL. They used HMM for sign language modeling. The accuracies for single character recognition and fingerspelt word recognition were $84.1 \%$ and $98.9 \%$ respectively. However, the latter accuracy was obtained when the HMM was trained using 100 words. If more words are added, the accuracy starts to fall. Rekha et al. [21] used a similar approach, although the domain of their work was much smaller. They covered 15 letters of ASL and 10 words formed by these letters.

Among the works based on data gloves, the reported best accuracy of $96 \%$ was obtained by Elmahgiubi et al. [11]. However, there are a few drawbacks of this work. Firstly, they left out six letters from the ASL alphabet including one dynamic letter. Although they covered the other dynamic letter 'J', they did not clarify the mechanism behind this. Secondly, they performed classification using a range-based query without clarifying how they came up with the ranges. Finally, sign language modeling was not covered in their work. Patil et al. [20] claimed to classify all the 26 letters of ASL using flex sensors only. Flex sensors can only detect bending, they did not clarify how they detected contact, orientation, and motion using only flex sensors. They did not provide any accuracy of their system, and just like the previous work, left out sign language modeling. Mehdi et al. [15] achieved 88\% accuracy over ASL alphabet using a commercially available glove from 5DT [2]. They left out the dynamic letters and sign language modeling just like the previous works. Vamplew et al. [22] worked on 52 gestures (including dynamic symbols) of Australian Sign Language (Auslan) using a commercially available glove from CyberGlove [1] and achieved an accuracy of $94 \%$. However, sign language modeling was not discussed by them either.

\section{CONCLUSION}

This work presents ASL and BdSL alphabet recognition using data gloves. Recognition of both dynamic and static character of ASL and BdSL is possible using the developed system. Sign language modeling has also been discussed in this work. Although sign language modeling using image processing technique has received some attention from the research community, not much work has been done for sign language modeling using data gloves. From that perspective, this work provides new insight into this problem. The lack of dataset creates hindrance in the analysis of data glove based systems. This work attempts to alleviate this problem by generating a dataset containing 1000 data points for each of the letters of ASL and BdSL. In the future, the work can be extended by improving the sign language modeling. A unified design of a data glove that can cover most of the different sign languages is another possible research direction. 
Table 4: Comparison with previous works.

\begin{tabular}{|c|c|c|c|c|c|c|c|}
\hline \multirow{2}{*}{ Author } & \multirow{2}{*}{ Language } & \multicolumn{2}{|c|}{ Symbol } & \multirow{2}{*}{ Method } & \multirow{2}{*}{ Algorithm } & \multicolumn{2}{|c|}{ Accuracy } \\
\hline & & $\begin{array}{l}\text { Letters left } \\
\text { out }\end{array}$ & $\begin{array}{l}\text { No. of finger- } \\
\text { spelt words }\end{array}$ & & & Recognition & Modeling \\
\hline Akmeliawati et al. [7] & Malayasian & - & - & DIP & ANN & $95.67 \%$ & - \\
\hline Kuznetsova et al. [13] & ASL & $\mathrm{J}, \mathrm{Z}$ & - & DIP & $\begin{array}{l}\text { multi-layered ran- } \\
\text { dom forest }\end{array}$ & $97.55 \%$ & - \\
\hline Dogic et al. [18] & Bosnian & - & - & DIP & ANN & $84.00 \%$ & - \\
\hline Dong et al. [10] & ASL & $\mathrm{J}, \mathrm{Z}$ & - & DIP & random forest & $90.00 \%$ & - \\
\hline Nabiyev et al. [16] & Turkish & 21 consonants & - & DIP & ANN & $96.00 \%$ & - \\
\hline Pandey et al. [19] & ASL & - & - & DIP & ANN & $90.00 \%$ & - \\
\hline Liwicki et al. [14] & BSL & - & 100 & DIP & $\begin{array}{l}\text { multi-class logis- } \\
\text { tic regression / } \\
\text { HMM }\end{array}$ & $84.10 \%$ & $98.90 \%$ \\
\hline $\begin{array}{l}\text { Naoum-Sawaya et } \\
\text { al. [17] }\end{array}$ & ASL & $\mathrm{N} / \mathrm{A}$ & $\mathrm{N} / \mathrm{A}$ & DIP & CAMSHIFT & $96.00 \%$ & - \\
\hline Rekha et al. [21] & ASL & $\begin{array}{l}\text { E,G,H,J,K,N, } \\
\mathrm{O}, \mathrm{P}, \mathrm{Q}, \mathrm{S}, \mathrm{T}, \mathrm{Z}\end{array}$ & 10 & DIP & KNN-SVM/HMM & $90.0 \%$ & $96.00 \%$ \\
\hline Patil et al. [20] & ASL & - & - & glove & ad-hoc & $\begin{array}{l}\text { not men- } \\
\text { tioned }\end{array}$ & - \\
\hline Elmahgiubi et al. [11] & ASL & E,M,N,S,T,Z & - & glove & ad-hoc & $96.00 \%$ & - \\
\hline Mehdi et al. [15] & ASL & $\mathrm{J}, \mathrm{Z}$ & - & glove & ANN & $88.00 \%$ & - \\
\hline Vamplew et al. [22] & Australian & $\mathrm{N} / \mathrm{A}$ & $\mathrm{N} / \mathrm{A}$ & glove & ANN & $94 \%$ & - \\
\hline
\end{tabular}

\section{ACKNOWLEDGMENTS}

This work was partially supported by the research grant under Higher Education Quality Enhancement Project (HEQEP) CP-3137 awarded by World Bank and approved by University Grant Commission (UGC), Bangladesh.

\section{REFERENCES}

[1] [n.d.]. CyberGlove Systems LLC. Available online at http://www. cyberglovesystems.com/. Last accessed 8:49 pm, August 05, 2017.

[2] [n.d.]. Data Gloves / 5DT. Available online at http://www.5dt.com/data-gloves/. Last accessed 8:51 pm, August 05, 2017.

[3] [n.d.]. How Much Do Sign Language Interpreters Get Paid? Available online at work chron.com/much-sign-language-interpreters-paid-8154.html. Last accessed 9:35 pm, August 04, 2017.

[4] [n.d.]. Sign Language: Fingerspelling. Available online at http://www.lifeprint com/asl101/pages-layout/fingerspelling.htm. Last accessed 6:32 pm, 04/24/2017.

[5] [n.d.]. Sign Language Recognition using Data Gloves. Available online at https: //saquib2527.github.io/slr.html\#dataset. Last accessed 3:24 pm, 12/19/2018.

[6] [n.d.]. WHO/Deafness and hearing loss. Available online at http://www.who.int/ mediacentre/factsheets/fs300/en/. Last accessed 9:08 pm, August 04, 2017.

[7] Rini Akmeliawati, Melanie Po-Leen Ooi, and Ye Chow Kuang. 2007. Real-time Malaysian sign language translation using colour segmentation and neural network. In Proceedings of the IEEE Instrumentation and Measurement Technology Conference (IMTC), Warsaw, Poland, May 1-3.

[8] Necati Cihan Camgoz, Simon Hadfield, Oscar Koller, Hermann Ney, and Richard Bowden. 2018. Neural sign language translation. In Proceedings of the IEEE Conference on Computer Vision and Pattern Recognition. 7784-7793.

[9] Runpeng Cui, Hu Liu, and Changshui Zhang. 2017. Recurrent convolutional neural networks for continuous sign language recognition by staged optimization. In Proceedings of the IEEE Conference on Computer Vision and Pattern Recognition. 7361-7369.

[10] Cao Dong, Ming C Leu, and Zhaozheng Yin. 2015. American sign language alphabet recognition using microsoft kinect. In Proceedings of the IEEE Conference on Comp. Vision and Patt. Recog. Workshops.

[11] Mohammed Elmahgiubi, Mohamed Ennajar, Nabil Drawil, and Mohamed Samir Elbuni. 2015. Sign language translator and gesture recognition. In Proceedings of the IEEE Global Summ. on Comp. \& Inf. Tech. (GSCIT), Sousse, Tunisia, June 11-13.

[12] Max Jaderberg, Karen Simonyan, Andrea Vedaldi, and Andrew Zisserman. 2014 Synthetic data and artificial neural networks for natural scene text recognition.
arXiv preprint arXiv:1406.2227 (2014)

[13] Alina Kuznetsova, Laura Leal-Taixé, and Bodo Rosenhahn. 2013. Real-time sign language recognition using a consumer depth camera. In Proceedings of the IEEE Int. Conf. on Computer Vision Workshops (ICCV), Sydney, Australia, December 1-2.

[14] Stephan Liwicki and Mark Everingham. 2009. Automatic recognition of fingerspelled words in british sign language. In Computer Vision and Pattern Recognition Workshops, 2009. CVPR Workshops 2009. IEEE Computer Society Conference on.

[15] Syed Atif Mehdi and Yasir Niaz Khan. 2002. Sign language recognition using sensor gloves. In Proceedings of the IEEE International Conference on Neural Information Processing (ICONIP), Singapore, November 18-22.

[16] VV Nabiyev and S Bayrak. 2006. An artificial neural network approach for sign language vowels recognition. In Proceedings of the Int. Scientific Conf. of Problems of Cybernetics and Informatics (PCI), Baku, Azerbaijan, October 24-26.

[17] Joe Naoum-Sawaya, Mazen Slim, Sami Khawam, and Mohamad Adnan Al-Alaoui. 2006. Dynamic system design for american sign language recognition. In Proceedings of ISCCSP.

[18] Sabaheta ogi and Gunay Karli. 2014. Sign Language Recognition using Neural Networks. TEM fournal 3, 4 (2014), 296-301.

[19] Pratibha Pandey and Vinay Jain. 2015. An efficient algorithm for sign language recognition. computer 6 (2015), 7.

[20] Kiratey Patil, Gayatri Pendharkar, GN Gaikwad, and Savitribai Phule. 2014. American sign language detection. International Journal of Scientific and Research Publications 4, 11 (2014).

[21] J Rekha, J Bhattacharya, and S Majumder. 2011. Hand gesture recognition for sign language: A new hybrid approach. In The 2011 International Conference on Image Processing, Computer Vision, and Pattern Recognition (IPCV).

[22] Peter Vamplew and Anthony Adams. 1996. Recognition of sign language gestures using neural networks. In Proceedings of the European Conf. on Disabilities, Virtual Reality and Associated Technologies (ECDVRAT), Maidenhead, UK, July 8-10.

[23] Tim Van den Bulcke, Koenraad Van Leemput, Bart Naudts, Piet van Remortel, Hongwu Ma, Alain Verschoren, Bart De Moor, and Kathleen Marchal. 2006. SynTReN: a generator of synthetic gene expression data for design and analysis of structure learning algorithms. BMC bioinformatics 7, 1 (2006), 43.

[24] Tamás Varga and Horst Bunke. 2003. Generation of synthetic training data for an HMM-based handwriting recognition system. In Document Analysis and Recognition, 2003. Proceedings. Seventh International Conference on. IEEE, 618-622. 